\title{
Sleep Respiratory Instability in Term Neonates under Hyperthermic Conditions: Age, Sex, Type of Feeding, and Rapid Eye Movements
}

\author{
ALFRED STEINSCHNEIDER ${ }^{(19)}$ AND STEVEN WEINSTEIN
}

The Sudden Infant Death Syndrome Institute, Department of Pediatrics, University of Maryland School of Medicine, Baltimore, Maryland, USA

\begin{abstract}
Summary
The purpose of this study was to examine the influence of neonatal age, sex, type of feeding, and rapid eye movements on the occurrence of apneic pauses during sleep in a hyperthermic environment. One hundred and twenty fullterm infants (equally divided by sex and type of feeding) were observed during a complete nap within the first and approximately fourth wk of life. Each sleep session was characterized by twenty-one measures including the longest apneic pause, mean apnea duration, relative frequency of apnea, apnea periodicity, respiratory rate, and relative frequency of REM epochs. The relative frequency of REM epochs decreased with age and was greater in formula-fed (versus breast-fed) infants.

The longest apneic pauses were greater in the first wk of life and in breast-fed infants. In addition, the average Longest Duration: $R E M \smile$ was $\smile$ greater $\smile$ than $\smile$ the $\smile$ average $\smile$ Longest Duration:NREM. Of potential importance was the demonstration that this latter effect was greater in the first wk than in the fourth wk of life. Breast-fed infants and females had larger apnea Mean Duration scores. The relative frequency of apneic pauses $(\geq 2 \mathrm{sec}$ in duration), periodicity, and the relative amount of apnea were greater in the fourth-wk study and in breast-fed infants. The relative frequency of apneic pauses $\geq 6 \mathrm{sec}$ in duration (Apnea 6 \%) and the $A_{6} / D \%$ measure were greater in breast-fed infants. Numerous interactions were observed between age, sex, and type of feeding. Respiratory rate decreased with age and was greater during NREM epochs and in formula-fed infants.
\end{abstract}

\section{Speculation}

The study of brief apneic pauses in the neonatal age period support the hypothesis that the mechanisms responsible for the initiation of apnea differs from those involved in its termination. It can be anticipated that abnormalities in either set of mechanisms might have pathologic consequences. The observations that apneic pauses are influenced by age, sex, type of feeding, and rapid eye movements indicate that careful consideration must be given to these variables when comparing infant groups. In addition these results have implications for increasing our understanding of respiratory control mechanisms, the development of prolonged sleep apnea and, theoretically, the occurrence of the sudden infant death syndrome.

This study is part of a programmatic effort designed to evaluate the hypothesis that prolonged sleep apnea is part of the pathophysiology resulting in the sudden infant death syndrome (SIDS) $(10,11)$ and was initiated to examine the affect on sleep apnea of variables known or presumed to influence the incidence of SIDS. In a previously published report (12) it was demonstrated that the frequency of reported episodes of prolonged sleep apnea increased during a nasopharyngitis. These data, obtained from infants ob- served at home on an apnea monitor, were interpreted as consistent with the sleep apnea-SIDS hypothesis; however, difficulty in controlling environmental variables and the numerous problems associated with the use of commercially available apnea monitors in the home (such as the frequency of false alarms as well as its potential psychologic effects on family members) limit its potential as a routine research technique for assessing the influence of SIDS-related variables on the occurrence of prolonged apnea.

The current study makes use of the observation that infants who have prolonged sleep apnea, in contrast to controls, also demonstrate more frequent and longer apneic pauses during a complete nap when observed in a controlled hyperthermic environment (13). In the latter study, this testing approach was employed because of the underlying hypothesis that respiratory instability was most apt to occur when infants were exposed either to an environmental or endogenous stress. The choice of a hyperthermic environment was based on the observation that prolonged apnea tended to occur when the ambient temperature was elevated (7). The primary purpose of this study was to examine the affects of neonatal age, sex, type of feeding, and rapid eye movements on the occurrence of apneic pauses during sleep. Because the occurrence of apneic pauses may be a reflection of a more generalized ability to control respiratory activity, it also seemed appropriate to examine the influence of these same variables on respiratory rate.

\section{MATERIALS AND METHODS}

One hundred and twenty full-term Caucasian infants with an average birth weight of $3481 \mathrm{~g}$ (range, 2608-5131 g) participated in this study. They were all clinically normal and born after an uneventful pregnancy and delivery. The 1- and 5-min Apgar scores were at least 8 . There were an equal number (60) of males and females and the same number of breast-fed and proprietary formula-fed infants in each of the two sex categories. All infants were studied in the sleep laboratory at two age periods. The initial study (age period 1) occurred within the first wk of life (range, 3 7 days; 106 of the subjects observed at age 3 days). The repeat study (age period 4) was performed, in general, within the fourth wk of life (range, 22-32 days; 107 subjects were observed between the age of 22-28 days). Written informed consent was obtained before the initial sleep study.

A complete nap was observed in an environmentally-controlled room with the ambient temperature set at $90 \pm 2^{\circ} \mathrm{F}$. Infants were brought to the laboratory from the nursery or home for a morning or afternoon nap. Surface electrodes were employed to record: (1) respiration-nasal: thermister taped below one nostril; (2) respiration-thorax: mercury strain gauge positioned across the lower thorax; (3) extraocular movements-vertical: flat disc electrodes placed above and below one eye; and (4) extraocular movementshorizontal: flat disc electrodes taped to the nasion and outer canthus. Gross motor activity was observed visually by an observer stationed outside the test room who viewed the infant through a 
Table 1. Laboratory sleep measures: definitions

\begin{tabular}{|c|c|c|}
\hline \multicolumn{2}{|c|}{ Sleep measure } & Longest apneic pause $(\mathrm{sec})$ \\
\hline Longest Duration & $\begin{array}{l}\text { Total (LT) } \\
\text { REM (LR) } \\
\text { NREM (LN) }\end{array}$ & $\begin{array}{l}\text { Longest apneic pause }(\mathrm{sec}) \\
\text { Longest apneic pause }(\mathrm{sec}) \text { initiated during a REM epoch } \\
\text { Longest apneic pause }(\mathrm{sec}) \text { initiated during a NREM epoch }\end{array}$ \\
\hline Mean Duration & $\begin{array}{l}\text { Total (MT) } \\
\text { REM (MR) } \\
\text { NREM (MN) }\end{array}$ & $\begin{array}{l}\text { Average duration (sec) of apneic pauses }(\geqslant 2 \mathrm{sec}) \\
\text { Average duration }(\mathrm{sec}) \text { of those apneic pauses }(\geqslant 2 \mathrm{sec}) \text { initiated during REM epochs } \\
\text { Average duration }(\mathrm{sec}) \text { of those apneic pauses }(\geqslant 2 \mathrm{sec}) \text { initiated during NREM epochs }\end{array}$ \\
\hline Apnea $_{2} \%$ & $\begin{array}{l}\text { Total (AT) } \\
\text { REM (AR) } \\
\text { NREM (AN) }\end{array}$ & $\begin{array}{l}\% \text { of epochs during which at least one apneic pause }(\geqslant 2 \mathrm{sec}) \text { was initiated } \\
\% \text { of REM epochs during which at least one apneic pause }(\geqslant 2 \mathrm{sec}) \text { was initiated } \\
\% \text { of NREM epochs during which at least one apneic pause }(\geqslant 2 \mathrm{sec}) \text { was initiated }\end{array}$ \\
\hline $\mathrm{A}_{2} / \mathrm{D} \%$ & & $\frac{\text { Duration of all apneic pauses }(\geqslant 2 \mathrm{sec})}{\text { Sleep Duration }} \times 100$ \\
\hline Periodic $_{2} \%$ & $\begin{array}{l}\text { Where periodic } \\
\text { pauses in the }\end{array}$ & $\begin{array}{l}\frac{\text { Number of epochs during which periodic apnea occurred }}{\text { Total number of epochs }} \\
\text { the occurrence of an apneic pause }(\geqslant 2 \mathrm{sec}) \text { in two successive epochs or two or more } \\
\text { och. }\end{array}$ \\
\hline REM\% & & $\frac{\text { Number of REM epochs }}{\text { Total number of epochs }} \times 100$ \\
\hline $\mathrm{PSA}_{4}$ & $-2.695+0.607$ & $0.023(\mathrm{AR})+0.042(\mathrm{AN})-0.143\left(\mathrm{~A}_{2} / \mathrm{D} \%\right)$ \\
\hline Apnea $6 \%$ & $\begin{array}{l}\text { Total } \\
\text { REM } \\
\text { NREM }\end{array}$ & $\begin{array}{l}\% \text { of epochs during which at least one apneic pause }(\geqslant 6 \mathrm{sec}) \text { was initiated } \\
\% \text { of REM epochs during which at least one apneic pause }(\geqslant 6 \mathrm{sec}) \text { was initiated } \\
\% \text { of NREM epochs during which at least one apneic pause }(\geqslant 6 \mathrm{sec}) \text { was initiated }\end{array}$ \\
\hline Periodic 6 & $\begin{array}{l}\text { Where periodic } \\
\text { pauses in the }\end{array}$ & $\begin{array}{l}\frac{\text { number of epochs during which periodic apnea occurred }}{\text { Total number of epochs }} \times 100 \\
\text { the occurrence of an apneic pause }(\geqslant 6 \mathrm{sec}) \text { in two successive epochs or two or more } \\
\text { och. }\end{array}$ \\
\hline $\mathrm{A}_{6} / \mathrm{D} \%$ & & $\frac{\text { Duration of all apneic pauses } ₹ 6 \mathrm{sec})}{\text { Sleep Duration }} \times 100$ \\
\hline
\end{tabular}

one-way vision mirror. All physiologic activity was recorded on a Grass 7 polygraph (Grass Instrument Co., Quincy, MA) with a gain setting of $50 \mu \mathrm{V} / \mathrm{cm}$ for the two eye movement channels and a paper speed of $10 \mathrm{~mm} / \mathrm{sec}$.

Each 15-sec epoch was identified as either a REM or NREM epoch based on the occurrence (or nonoccurrence) of rapid eye movements. The polygraphic tracing was examined visually and all apneic pauses (no activity in both respiratory channels) at least $2 \mathrm{sec}$ in duration were measured to a tenth of a sec. The sleep session was characterized by 21 measures including the longest apneic pause, mean apnea duration, relative frequency of apnea, apnea periodicity, and relative frequency of REM epochs (see Table 1 for definitions). Table 1 also contains the equation employed in calculating the $\mathrm{PSA}_{4}$ (defined by equation in Table 1) measure of respiratory instability (13). The Mean Duration (Total, REM, NREM), Apnea $2 \%$ (Total, REM, NREM), Periodic ${ }_{2} \%, \mathrm{~A}_{2} /$ $\mathrm{D} \%$ and $\mathrm{PSA}_{4}$ measures were based on apneic pauses $\geq 2 \mathrm{sec}$ in duration. Only apneic pauses at least $6 \mathrm{sec}$ in duration were used in calculating the Apnea 6 (Total, REM, NREM), Periodic ${ }_{6} \%$ and $\mathrm{A}_{6} \mathrm{D} \%$ measures.

The average respiratory rate was obtained for REM and NREM epochs separately. Thirty REM epochs free of apneic pauses $(\geq 2$ sec) were chosen by employing a table of random numbers, and the number of completed or near completed (75\% of cycle) respirations counted within each $15-\mathrm{sec}$ epoch. The average was computed for REM epochs and multiplied by four. The same procedure was employed in obtaining the average respiratory rate during NREM epochs. This approach was chosen to avoid confounding measures of respiratory rate with those of apnea.

The data were analyzed statistically employing an analysis of variance model. A $\log _{10}$ transformation was employed on the Apnea $_{2} \%$ (Total, REM, NREM), Periodic $2 \%, \mathrm{~A}_{2} / \mathrm{D} \%$, Apnea 6 (Total, REM, NREM), Periodic $6 \%$ and $\mathrm{A}_{6} \mathrm{D} \%$ measures before conducting the analysis of variance. A three-factor analysis of variance (age, sex, type of feeding) was conducted on each of the measures except those related to either REM or NREM epochs. A four-way analysis of variance (age, sex, type of feeding, sleep epoch) was used for these latter measures (Longest Duration:REM, NREM; Mean Duration:REM, NREM; Apnea $_{2} \%$ REM; Apnea $\%:$ NREM; Apnea $\%$ REM; Apnea $\%$ : NREM; Respiratory Rate:REM; and Respiratory Rate:NREM). Separate analyses of variance were conducted for breast-fed infants and for infants receiving formula as a means of clarifying significant interactions involving type of feeding. An F-ratio was considered statistically significant when the resultant $P$-value was $\leq 0.05$.

\section{RESULTS}

Table 2 contains the average results obtained for all sleep measures as a function of type of feeding (breast, formula), sex (male, female), and age period $(1,4)$. Each average is based on 30 subjects.

Sleep characteristics. Sleep duration averaged $110 \mathrm{~min}$ and was not influenced either by sex or type of feeding. Infants did sleep significantly longer during the first wk sleep study $(P<0.01)$. The average sleep durations were $120.4 \mathrm{~min}$ and $99.6 \mathrm{~min}$ during the first and fourth wk sleep study respectively.

The relative frequency of REM epochs was influenced significantly by both postnatal age $(P<0.01)$ and type of feeding $(P<$ 
Table 2. Average value for each sleep measure

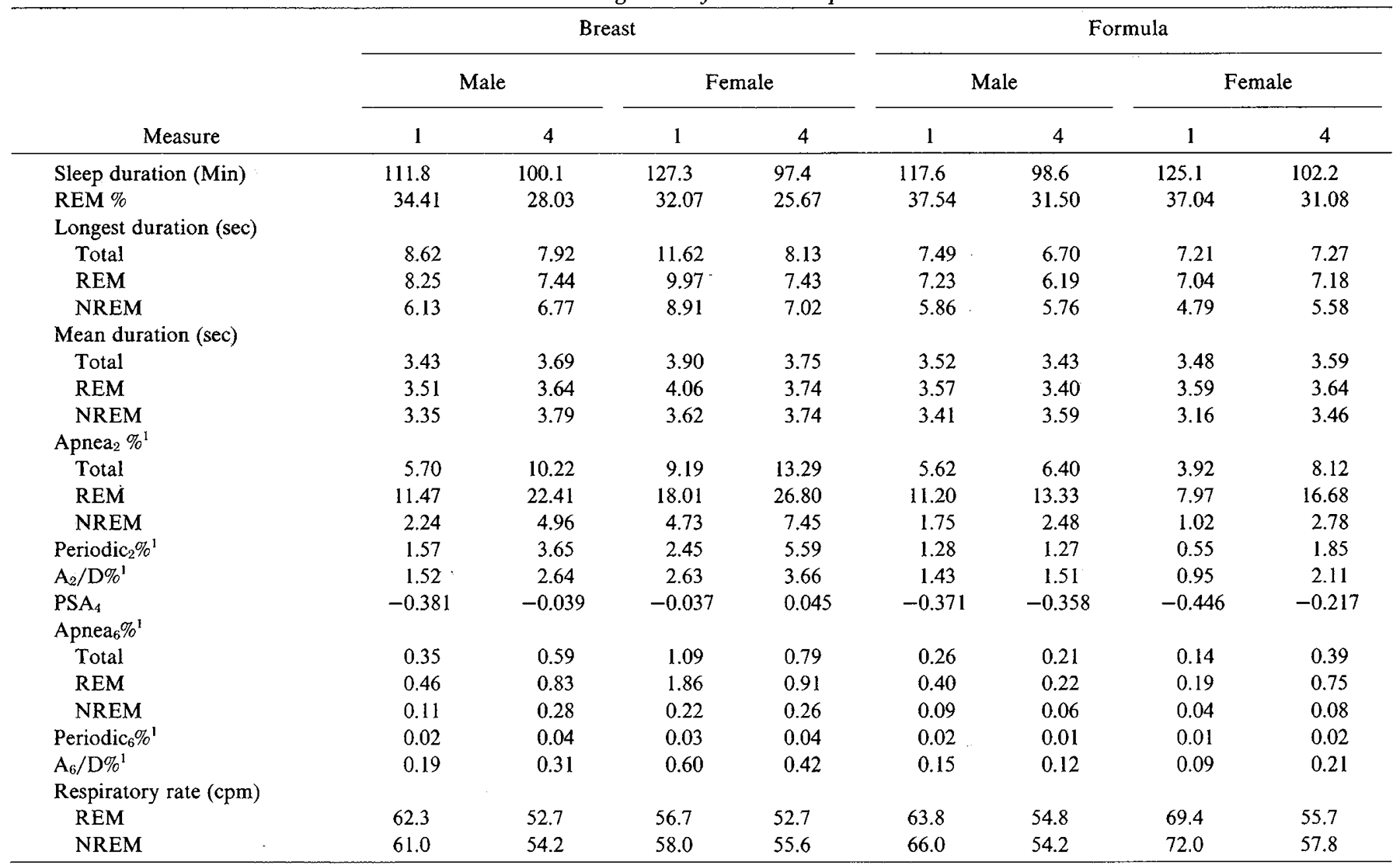

${ }^{1}$ Antilog.

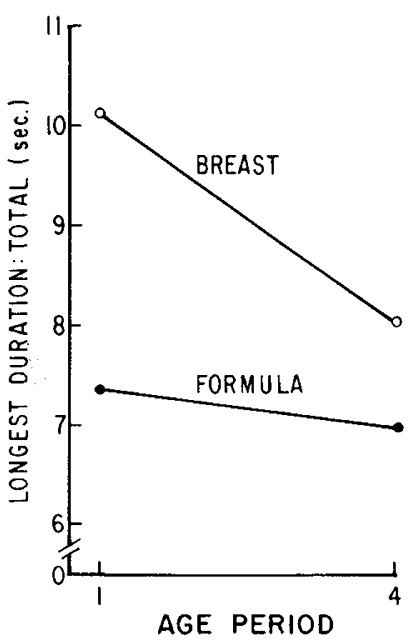

Fig. 1. The influence of age and type of feeding on the Longest Duration:Total.

$0.01)$. During age period $1,35.3 \%$ of epochs were associated with rapid eye movements whereas there were $29.1 \%$ REM epochs during age period 4 . Breast-fed infants had significantly fewer REM epochs (30.1\%) when compared to infants receiving a proprietary formula (34.4\%). Sex was not found to affect the relative frequency of REM epochs.

Apneic pause. In general, the longest apnea duration (Longest Duration:Total) was influenced by postnatal age $(P<0.01)$ and type of feeding $(P<0.01)$. Longer apneic pauses were observed within the first wk of life and in breast-fed infants. On the average, the longest apnea was $8.7 \mathrm{sec}$ during the first wk and $7.5 \mathrm{sec}$ within the fourth wk. The longest apnea averaged $9.1 \mathrm{sec}$ in breast-fed infants and $7.2 \mathrm{sec}$ in formula-fed infants; however, the interpre-

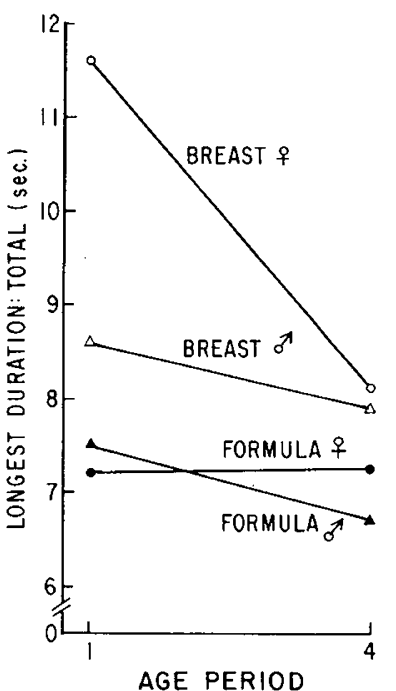

Fig. 2. The influence of age, type of feeding, and sex on the Longest Duration:Total

tation of these data is complicated by the observation that the effect of age is influenced both by sex and the type of feeding $(P$ $<0.05$; Fig. 1 and 2). For example, the difference between the feeding groups was greater in the first wk than in the fourth wk (Fig. 1). Furthermore an analysis of variance employing only formula-fed infants failed to demonstrate any significant effect of age of testing, sex or their interaction (Fig. 2). This contrasts with the analysis of variance performed on the breast-fed infants, which revealed not only that the longest apnea duration decreased significantly with age $(P<0.01)$ but that the magnitude of the sex effect was different at the two ages $(P<0.05)$. (In the first wk 
study, breast-fed females had longer pauses when compared to breast-fed males. Although a similar relationship was observed in the fourth wk, the difference between the sexes was much less). The analysis incorporating the longest pauses during REM and NREM (Longest Duration:REM and Longest Duration:NREM) revealed the same significant main and interaction effects as those observed when sleep epoch was not considered. In addition, the longest pause during REM was significantly greater than the longest pause during $\operatorname{NREM}(P<0.01)$. Of potential importance was the demonstration that the effect of rapid eye movements differed at the two ages $(P<0.05)$. Figure 3 reveals a greater difference between REM and NREM epochs at wk 1 than at wk 4 with little change between the two age periods for the NREM epochs.

Breast-fed infants $(P<0.01)$ and females $(P<0.05)$ had larger Mean Duration:Total scores compared to formula-fed infants and males, respectively. In addition the difference between the sexes was found to be influenced by type of feeding and age $(P<0.01)$. An analysis of variance performed only on formula-fed infants failed to demonstrate a significant effect of sex, age of testing, or sex by age interaction. By contrast breast-fed females, in general, had larger values than breast-fed males $(P<0.05)$. Furthermore, the effect of age in males differed from that in females $(P<0.05)$. Examination of Figure 4 (breast-fed subjects only) reveals that increasing age is associated with a decrease in the average duration for females, but an increase in males. (The average durations were almost the same for both sexes in age period 4). The analysis employing sleep epochs revealed similar relationships. Although REM epochs were associated with longer duration apneic pauses

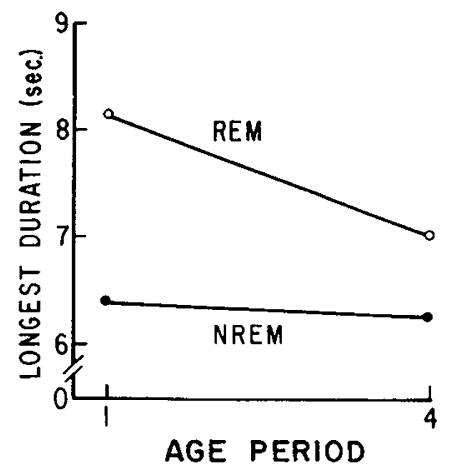

Fig. 3. The average Longest Duration:REM and NREM at each of the age periods.

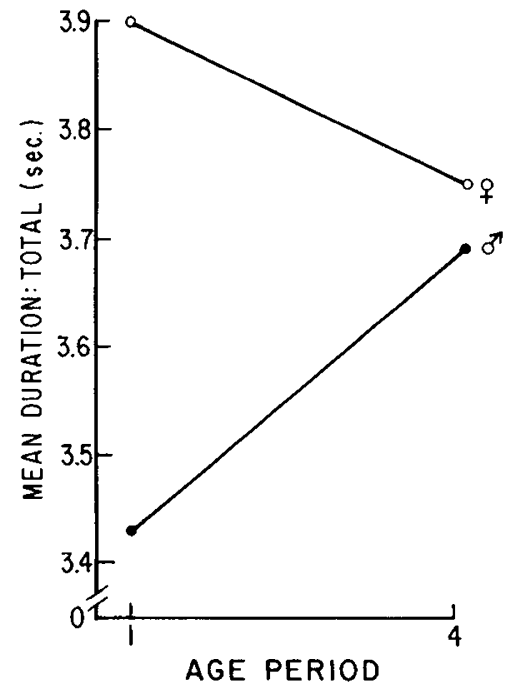

Fig. 4. The average Mean Duration:Total for males and females at each age period.

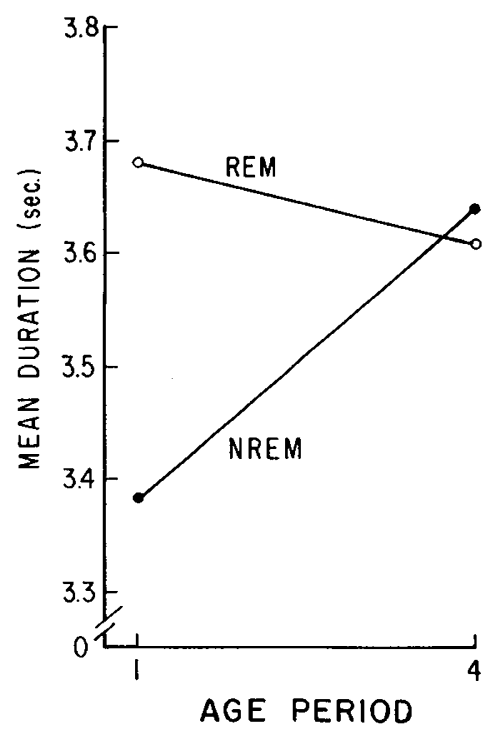

Fig. 5. The average Mean Duration:REM and NREM at each age period.

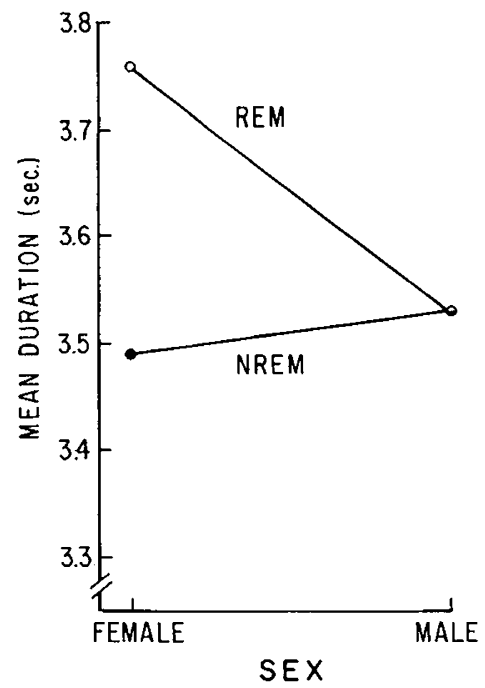

Fig. 6. The average Mean Duration:REM and NREM for males and females.

than NREM epochs $(P<0.01)$ the effect of sleep epoch was found to be influenced by both the age and sex of the infant (sleep stage by age interaction, $P<0.01$ and sleep stage by sex interaction, $P$ $<0.01$ ). Figure 5 indicates that REM epochs were associated with longer apneic pauses at wk 1 but were little, if any, different from NREM epochs at wk 4. Examination of Figure 6 reveals that the average duration during REM is greater than during NREM in females but not in males.

The relative frequency of apneic pauses as measured by Apnea $_{2} \%$ :Total was significantly greater $(P<0.01)$ in the fourth wk study (versus week 1) and in breast-fed (versus formula-fed) infants (Fig. 7). Similar relationships were observed when examining the Apnea ${ }_{2} \%:$ REM and NREM measures. In addition, relatively more apneic pauses were observed during REM epochs $(P<0.01)$. Although REM epochs (versus NREM epochs) were associated with more frequent apneic pauses regardless of the type of feeding, the difference between the different sleep epochs was greater in breast-fed than in formula-fed infants $(P<0.01$; Fig. 8).

Both Periodic ${ }_{2} \%$ and $\mathrm{A}_{2} / \mathrm{D} \%$ increased between age periods 1 and $4(P<0.01)$ and were greater in breast-fed infants $(P<0.01)$. The type of feeding and sex of the infant also influenced the effect of age on the $\mathrm{A}_{2} / \mathrm{D} \%$ measure $(P<0.05)$. More detailed analyses 
revealed that the increase in $\mathrm{A}_{2} / \mathrm{D} \%$ associated with age was greater in formula-fed females than in formula-fed males $(P<$ 0.05 ), whereas sex was not a factor on the age effect in breast-fed infants; however, in general, breast-fed females had significantly greater $\mathrm{A}_{2} / \mathrm{D} \%$ scores than breast-fed males $(P<0.05$, Table 2$)$.

The $\mathrm{PSA}_{4}$ measure was affected by postnatal age $(P<0.01)$, sex $(P<0.05)$ and type of feeding $(P<0.01)$. In general, more positive $\mathrm{PSA}_{4}$ scores were found in the fourth postnatal wk, breastfed infants and in females. The analysis of variance performed on the formula group revealed only a significant $(P<0.05)$ age effect (wk $4>$ wk 1). A similar analysis performed on breast-fed infants revealed both a significant age $(P<0.01)$ and sex $(P<0.05)$ effect (Fig. 9). Breast-fed infants also demonstrated a significantly different age effect for males and females $(P<0.05)$. Based on these additional analyses, it would appear that the $\mathrm{PSA}_{4}$ measure increases with age in both formula-fed and breast-fed infants; however, sex appears to be a relevant variable only in the breast-fed infants, with females having larger scores at both age periods.

The remaining apnea measures are all based on apneic pauses $\geq 6$ seconds in duration. Breast-fed infants had larger Apnea $\%$ : Total scores than formula-fed infants $(P<0.01)$. This measure was not significantly influenced by either sex or age in infants who were breast-fed. In formula-fed female infants significantly more frequent apneic pauses occurred in wk 4 (versus wk 1), whereas the reverse was true in formula-fed males $(P<$ 0.05 , see Table 2). Similar results were obtained when analyzing the Apnea $6:$ REM, Apnea $\%:$ NREM and the $\mathrm{A}_{6} / \mathrm{D} \%$ measures. In addition, REM epochs were associated with more frequent

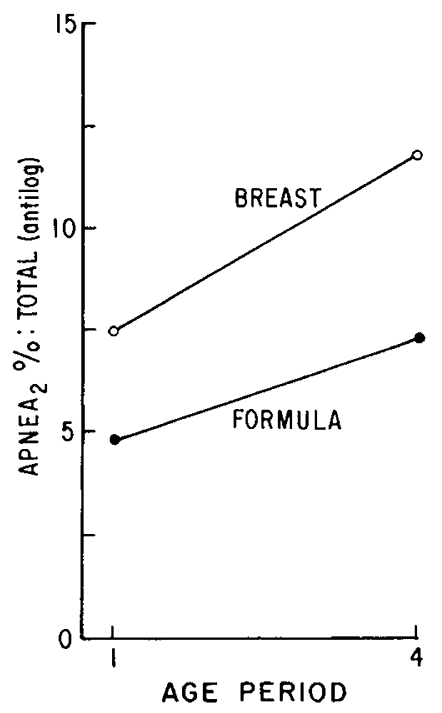

Fig. 7. The influence of age and type of feeding on Apnea $2 \%$ :Total.

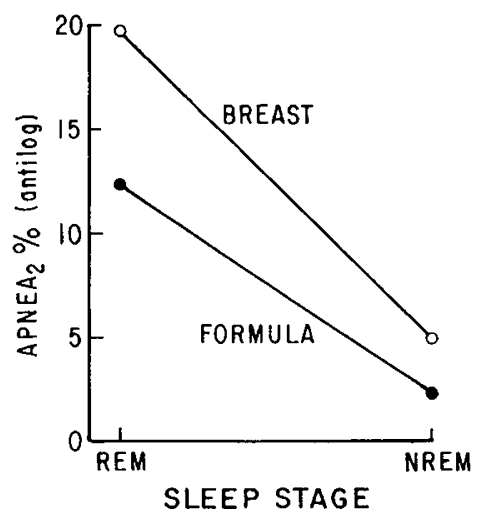

Fig. 8. The average Apnea $2 \%:$ REM and NREM in breast- and formulafed infants.

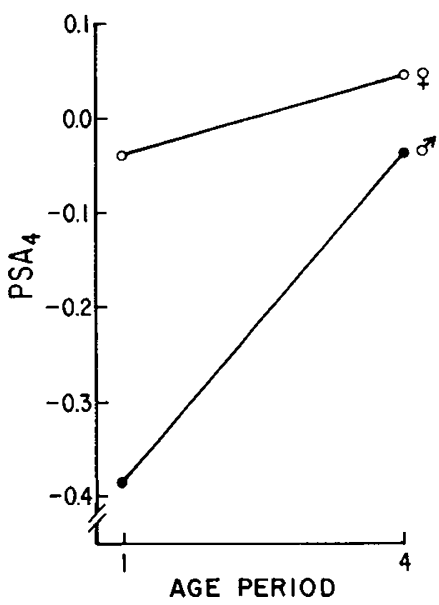

Fig. 9. The influence of age and sex on $\mathrm{PSA}_{4}$ scores in breast-fed infants.

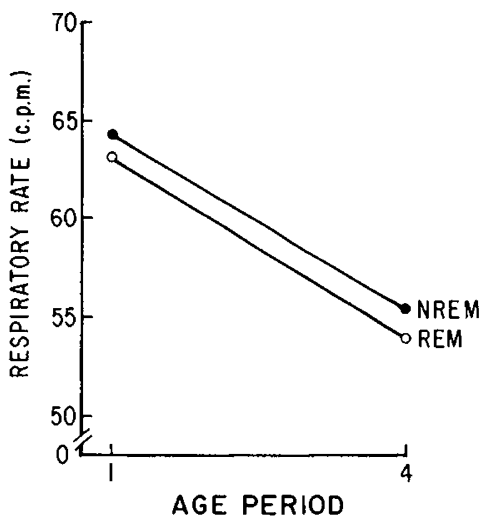

Fig. 10. The average respiratory rate during REM and NREM epochs at each age period.

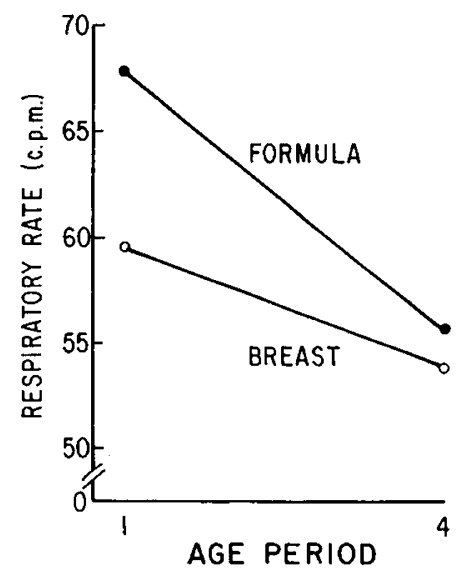

Fig. 11. The average respiratory rate in breast- and formula-fed infants at each age period.

apneic pauses $(P<0.01)$. The Periodic 6 measure was not influenced by either sex or age, though breast-fed infants had significantly more periodic apnea than did formula-fed infants $(P<$ 0.01).

Respiratory rate. The average respiratory rate in this study was 59.2 cycles/min. Respiratory rate was significantly greater in the first wk of life than in the fourth $(P<0.01)$, during NREM epochs $(P<0.05$; Fig. 10) and in formula-fed infants $(P<0.05)$. Furthermore, this latter effect was greater in wk 1 than in wk $4(P<$ 0.05 , Fig. 11). 


\section{DISCUSSION}

The results of this study demonstrate that the type of feeding, age of testing, sex, and occurrence of rapid eye movements all influence respiratory activity during sleep.

In general, breast-fed neonates demonstrated more frequent and longer apneic pauses compared to formula-fed babies. Additionally, the average respiratory rate was lower in breast-fed neonates. It is important to recognize that this latter observation cannot be attributed to the more frequent apneic pauses noted in breast-fed babies because respiratory rate was measured only in those epochs free of apneic pauses.

The research by Moore et al. (6) and Raiha et al. (8) provide a basis for understanding the differences in respiratory activity between formula-fed and breast-fed neonates. These investigations demonstrated that a cow's milk based formula induces a metabolic acidosis. One of the consequences of this metabolic acidosis could be stimulation of the respiratory centers resulting in an increase in respiratory rate and a decrease in the frequency of apneic pauses.

These observations have potential implications for the occurrence of prolonged sleep apnea and theoretically the sudden infant death syndrome. In view of the studies demonstrating an association between brief apneic pauses and the occurrence of prolonged apnea during sleep (13) it might be anticipated that infants fed human breast milk would be more likely to develop prolonged sleep apnea. In addition, based upon the hypothesis implicating a relationship between prolonged sleep apnea and SIDS (10), it might also be expected that breast-fed infants would be at increased risk for SIDS. No data bearing on the former theoretical prediction are available. Furthermore, in spite of considerable interest and discussion there is insufficient evidence to justify any definitive conclusions regarding the influence of human breast milk (versus proprietory formula based on cow's milk) on SIDS $(16,17)$. To test this latter theoretical prediction it would be necessary to control, among other variables, for the apparent protective effect of human breast milk against infection and the association between respiratory infection and SIDS.

Consistent with other published reports increasing age was associated with a decreasing \% of REM epochs and respiratory rate $(4,9)$. In addition, by the end of the first month of life, there was an increase in the frequency, periodicity, $\mathrm{A} / \mathrm{D} \%$, and $\mathrm{PSA}_{4}$ measures based on apneic pauses $\geq 2 \mathrm{sec}$ in duration; however, the average duration measures as well as those measures based on apneic pauses $\geq 6 \mathrm{sec}$ (Apnea $\%$ :Total, REM, NREM; Periodic 6 \%; $\mathrm{A}_{6} / \mathrm{D} \%$ ) were essentially the same at the two age periods investigated. These results are, in part, at variance with those obtained by Gould $e t$ al. (2) and Hoppenbrouwers et al. (5). Gould et al. (2) observed a decrease in the apnea index (a measure similar to the $\mathrm{A}_{2} / \mathrm{D} \%$ ) between $40-44 \mathrm{wk}$ postconceptual age for indeterminate sleep and no change over this same age period during REM and quiet sleep. This conflicts with the increase in the $\mathrm{A}_{2} / \mathrm{D} \%$ observed in the present study. Hoppenbrouwers et al. (5) found a decrease in apnea density (a measure similar to the Apnea 6 scores) during active sleep across the first 3 months of life and no significant age effect during quiet sleep. These discrepancies are difficult to resolve in view of the many methodologic differences between the various studies (e.g., hyperthermic versus normothermic environmental condition). Nonetheless, the results obtained in this study are supportive of the general hypothesis that important maturational changes affecting respiratory activity do occur within the first few months of life. Furthermore, the finding that the frequency measures (Apnea ${ }_{2} \%$ ) are affected differently by age than are the average duration scores (Mean Duration) supports the hypothesis that the mechanisms initiating apnea differ from those which limit their duration $(2,11)$.

The results obtained on the influence of sex also support this latter hypothesis. Regardless of type of feeding, females had longer average apneic pauses than did males. This contrasts with the failure to find a sex effect, per se, in the frequency measures; however, Thoman et al. (15) obtained results that differ from those of the present study. These investigators observed a greater fre- quency of apneic pauses in females but no significant effect of sex on the average apnea length (although the longest duration apneas occurred in females). Differences in methodology could account for the discrepancies between studies. The Thoman et al. study was conducted in the home (except for the first wk), employed a pressure transducer placed beneath the baby to record respirations, and used behavioral criteria to define each epoch. No information is provided on the infants feeding history nor was any apparent effort made to control for ambient temperature or the level of environmental stimulation. Furthermore, the influence of sex appears to be much more complicated than is reflected in the general effect because there is a complex interaction between sex, type of feeding, and age. In formula-fed infants, the increase in the frequency measures observed with age was greater in females. No sex by age interaction was observed in the duration measures. In breast-fed infants there was a significant interaction for the duration measures but no such interaction for the frequency measures. The attempt to explain this complex interaction within a general theoretical framework should await its confirmation. Nonetheless, in view of the observation that infants who have episodes of prolonged sleep apnea also demonstrate, in a sleep laboratory, more frequent and longer apneic pauses (13) it seems appropriate to predict on theoretical grounds that within the first month of life females would be more likely to develop prolonged sleep apnea and, theoretically, SIDS. Unfortuantely, no studies have been published examining the affect of sex on prolonged sleep apnea. In apparent contradiction with this prediction are the numerous reports demonstrating a greater incidence of SIDS in males (16). Importantly, these SIDS studies included infants up to at least 1 year of age and therefore are not totally comparable with the current study. Of greater relevance is the report by Fedrick (1) who found no sex difference in the incidence of SIDS when the death occurred within the first 11 wk of age; the male predominance was evident beyond this age period. This issue could be clarified by an intensive study which examines the affect of sex on the incidence of SIDS as a function of age of death.

The observation that REM epochs are associated with more frequent and longer apneic pauses has been made in several other studies $(2,5,10,13)$ and is once again confirmed. Similar results have prompted Gould et al. (2) to propose that the inhibitory affects of the REM state act at the level of the medulla inducing frequent apneic pauses ("apnea turn on") as well as longer pauses ("apnea turn off"). The results reported here appear to differ from those of Guilleminault et al. (3) who have concluded that the longest apneic episodes are most likely to occur not during REM but rather during NREM sleep. This conclusion was based on observations made on infants most of whom were first studied in the postneonatal age period. It is possible therefore that the difference in findings could be attributed to the age of the infants studied. This hypothesis receives support from the data presented by Gould et al. (2) and Waite and Thoman (18) who studied infants in a normothermic environment, as well as the observation in the present study that the affect of rapid eye movements on the longest apnea duration measure was different at the two ages. Although longer episodes occurred during REM epochs at both age periods studied, the differential affect of sleep epochs was greatest within the first wk of age.

The consistency with which slower respiratory rates has been found to be associated with NREM or quiet sleep $(5,9)$ and the observation in the current study that slower respiratory rates occurred during REM epochs raises questions about the comparability of epoch definition between this and other studies. Because one of the methodologic differences that existed between the studies was the ambient temperature of the test room, a subsequent study was conducted to examine for the possible influence of this latter variable (14). Employing the same general methodology used in the present study, REM epochs were found to be associated with more rapid respirations when neonates slept in a test room kept at $75^{\circ} \mathrm{F}$, whereas the respiratory rate was slower during REM epochs when the ambient room temperature was maintained at $90^{\circ} \mathrm{F}$. It would appear that the conflicting results can be due to 
differences in the environmental conditions under which the observations were made.

Previous reports have pointed out the importance of controlling for age, sex, and sleep state when examining sleep physiology. Type of feeding should be added to this list.

\section{REFERENCES AND NOTES}

1. Fedrick, J.: Sudden unexpected death in infants in the Oxford Record Linkage Area: An analysis with respect to time and place. Brit. J. Prev. Soc. Med., 27: 217 (1973).

2. Gould, J. B., Lee, A. F. S., James, O., Sander, L., Teager, H., and Fineberg, N.: The sleep state characteristics of apnea during infancy. Pediatrics, 59: 182 (1977).

3. Guilleminault, C., Peraita, R., Souquet, M., and Dement, W. C.: Apneas during sleep in infants: possible relationship with sudden infant death syndrome. Science, 190: 677 (1975)

4. Hoppenbrouwers, T., Harper, R. M., Hodgman, J. E., Sterman, M. B., and McGinty, D. J.: Polygraphic studies of normal infants during the first six months of life. II. Respiratory rate and variability as a function of state. Pediatr. Res., 12: 120 (1978).

5. Hoppenbrouwers, T., Hodgman, J. E., Harper, R. M., Hofmann, E., Sterman, M B., and McGinty, D. J.: Polygraphic studies of normal infants during the first six months of life. IIl. Incidence of apnea and periodic breathing. Pediatrics, 60: 418 (1977).

6. Moore, A., Ansell, C., and Barrie, H.: Metabolic acidosis and infant feeding. Brit. Med. J., $1: 129$ (1977)

7. Peristein, P. H., Edwards, N. K., and Sutherland, J. M.: Apnea in premature infants and incubator air temperature changes. N. Engl. J. Med., 272: 461 (1970).

Copyright $(1983$ International Pediatric Research Foundation, Inc. $0031-3998 / 83 / 1701-0035 \$ 02.00 / 0$
8. Raiha, N. C. R., Heinonen, K., Rassin, D. K., and Gaull, G. E.: Milk protein quantity and quality in low birthweight infants: Metabolic responses and effects on growth. Pediatrics, 57: 659 (1976).

9. Roffwarg, H. P., Muzio, J. N., and Dement, W. C.: Ontogenetic development of the human sleep-dream cycle. Science, 152: 604 (1966).

10. Steinschneider, A.: Prolonged apnea and the sudden infant death syndrome: Clinical and laboratory observations. Pediatrics, 50: 646 (1972).

11. Steinschneider, A.: Implications of the sudden infant death syndrome for the study of sleep in infancy. In: A. D. Pick, Ed.: Minnesota Symposia on Child Psychology, Vol. 9, pp. 106-134 (Minneapolis, University of Minnesota Press, 1975).

12. Steinschneider, A.: Nasopharyngitis and prolonged sleep apnea. Pediatrics, 56: 967 (1975).

13. Steinschneider, A.: Prolonged sleep apnea and respiratory instability: A discriminative study. Pediatrics, 59 (suppl): 962 (1977).

14. Steinschneider, A. and Weinstein, S. L.: Unpublished observations

15. Thoman, E. B., Freese, M. P., Becker, P. T., Acebo, C., Morin, V. N., and Tynan, W. D.: Sex differences in the ontogeny of sleep apnea during the first year of life. Physiol. Behav., 20: 699 (1978).

16. Valdes-Dapena, M. A.: Sudden unexplained infant death, 1970 through 1975: an evolution in understanding. Pathol. Annu., 12: 117 (1977).

17. Valdes-Dapena, M. A.: Sudden infant death syndrome: a review of the medical literature 1974-1979. Pediatrics, 66: 597 (1980)

18. Waite, S. P. and Thoman, E. B.: Brief apneas and reliable assessment of respiratory instability. Sleep, 4: 61 (1981).

19. Requests for reprints should be addressed to: Dr. Alfred Steinschneider, Director, The Sudden Infant Death Syndrome Institute, Baltimore, MD 21201.

20. This research was supported in part by research grants HD 07460 and HD 10982 from the National Institute of Child Health and Human Development.

21. Received for publication August 19, 1981

22. Accepted for publication May 6, 1982. 